\title{
Levels of Physical Activity, Obesity and Related Factors in Young Adults Aged 18-30 During 2009-2017
}

\author{
José Alberto Laredo-Aguilera ${ }^{1,2,3}$ (D), Ana Isabel Cobo-Cuenca $1,2,3, * \mathbb{C}$, \\ Esmeralda Santacruz-Salas 1,2 , María Manuela Martins ${ }^{4}$, María Aurora Rodríguez-Borrego 3,5,6, \\ Pablo Jesús López-Soto ${ }^{3,5,6}$ and Juan Manuel Carmona-Torres 1,2,3 (D) \\ 1 Department of Nursing, Physiotherapy and Occupational Therapy, Universidad de Castilla-La Mancha, \\ 45600 Talavera de la Reina, 45071 Toledo, Spain; jalaredo@hotmail.com (J.A.L.-A.); \\ esmeralda.santacruz@uclm.es (E.S.-S.); juanmanuel.carmona@uclm.es (J.M.C.-T.) \\ 2 Grupo de Investigación Multidisciplinar en Cuidados (IMCU), 45071 Toledo, Spain \\ 3 Instituto Maimónides de Investigación Biomédica de Córdoba (IMIBIC), 14004 Córdoba, Spain; \\ en1robom@uco.es (M.A.R.-B.); n82losop@uco.es (P.J.L.-S.) \\ 4 Escola Superior de Enfermagem/CINTESIS, 4200-072 Porto, Portugal; mmartins@esenf.pt \\ 5 Universidad de Córdoba, 14004 Córdoba, Spain \\ 6 Hospital Universitario Reina Sofía de Córdoba, 14004 Córdoba, Spain \\ * Correspondence: anaisabel.cobo@uclm.es; Tel.: +34-92-526-8800 (ext. 5818)
}

Received: 20 September 2019; Accepted: 19 October 2019; Published: 21 October 2019

check for updates

\begin{abstract}
The objective of this study was to analyze the temporal trend of physical activity and body mass index in young adults aged 18-30 in Spain and to ascertain their relationship with sociodemographic and psychosocial variables in the period of 2009-2017. Methods: A descriptive study with a sample of 10,061 young adults aged 18-30 years was performed. The data were obtained from the European Health Survey in Spain in 2009 and 2014 and the National Health Survey in 2011/2012 and 2017. The chi-square test was used for qualitative variables, and multiple linear regression analysis was performed for physical activity. Results: Sedentary levels had decreased in 2017 as compared to 2011/2012 $(p<0.001)$; smokers were more sedentary than non-smokers $(p<0.001)$; men were more active than women $(p<0.001)$; and the year with the highest physical activity was 2014 . Body mass index in the total sample increased from 2009 to $2017(p<0.01)$, showing a significant increase in obesity in women $(p<0.05)$ and no difference in men $(p \geq 0.05)$. Conclusions: In the period 2011/2012-2017, the sedentary lifestyle of young adults was reduced and physical activity was increased, with men being more active than women.
\end{abstract}

Keywords: young adults; physical activity; obesity; overweight; BMI

\section{Introduction}

There is increasing evidence that physical activity (PA) has very beneficial effects for people of all ages, and the sooner these habits are acquired, the greater the benefits, contributing to maintenance of a person's health as they advance in age [1].

Among different health disorders, there is obesity. Currently, $70 \%$ of adults with obesity have acquired it in adulthood [2]. On the other hand, anxiety and depression have been directly related to weight gain in young adults [3]. In Spain, the population aged 18-30 years amounts to 5.3 million [4], which gives an idea of the magnitude of the problem. Body mass index (BMI) and obesity are related to levels of PA [5].

Mechanization in all areas has affected the way of life, both in the workplace (reducing energy expenditure) and at home (increasing sedentary lifestyle) [6]. Sedentary behaviors, 
such as passive transport, have also been associated with increased obesity [7]. Physical activity is also important as an appetite regulator, observing that people with low levels of PA have higher levels of food cravings than people with high levels of PA [8]. It has been proven that both levels of PA and sociodemographic and psychosocial characteristics influence obesity [9]. In a 32-year longitudinal study, it was observed that twins with different lifestyles and sociodemographic and psychosocial differences had significant differences in body weight, BMI and fat percentage, with the lowest results in those that had the highest levels of PA [9].

A reduction in mobility is associated with lower levels of PA and higher mortality from cardiovascular disease [10]. The prevention of coronary heart disease requires a better understanding of risk factors and healthy lifestyle behaviors from an early age [11]. Several epidemiological studies [11-13] reflect that there are cardiovascular benefits and a decrease in the risk of cardiovascular diseases and deaths when practicing the recommended levels of PA [11]. These same authors [12,13] demonstrated that greater intensity and higher activity sessions have an association with reductions in risk and mortality from vascular diseases in white men.

According to Haskell et al. [14], PA in healthy people should be $150 \mathrm{~min}$ at moderate intensity or 60 mins at high intensity per week for health maintenance. Furthermore, the activity has to be for periods of $10 \mathrm{~min}$ at a minimum [14].

Andersson et al. [15] noted that maintaining recommended levels of PA showed a reduction of 1.8 years in vascular ageing. On the other hand, it is estimated that if adults reduced their sitting by $3 \mathrm{~h}$ or more per day, it would increase their life expectancy by two years [16]. Shah et al. [17] noted that every minute that PA was reduced in young adults over a period of seven years was significantly associated with cardiovascular disease and long-term mortality risk, but, if physical capacity was increased, heart failure was reduced. Other authors have emphasized that maintaining PA habits improves health status over the years and favors active ageing [18].

Previously, obesity has been mentioned as a health problem that can arise as a result of a decrease in PA [5]. Obesity is, in turn, an added health complication in people with chronic diseases and inflammatory processes [19].

The prevalence of overweight/obesity and its concomitant health risks are almost universal [20]. The World Health Organization aims to stop its increase by 2025 [21]. For this, it is necessary to quantify levels of overweight and obesity and to develop action policies in those places where it is most required [22].

Obesity has not decreased significantly in any country worldwide in the last 33 years [20]. However, it is expected that developed countries have reached the maximum point, while obesity in developing countries will not increase by more than $40 \%$; the truth, however, is that obesity is continuing to increase [20].

Another health disorder directly or indirectly related to decreased exercise is problems with sleep $[23,24]$. Problems including alterations in sleep quality or quantity affect up to $40 \%$ of young adults [23] and have an inverse association with sedentary lifestyle and obesity in young adults [24]. Increasing hours of sleep on weekends compared to sleep time during the week is associated with an increase in BMI and adiposity [5].

Physical inactivity and poor dietary intake or poor dietary habits are related to alterations in wellbeing, weight and health in general [25], all of which are associated with risks of ischemic heart disease, stroke, type 2 diabetes, depression and some types of cancer [25].

Another study [18] confirms the effects of PA levels, sedentary lifestyle, overweight and obesity, and the importance of maintaining PA habits in adulthood and in older adults.

The increase in rates of overweight and obesity in the young population [16], together with the health problems they cause during adulthood [15], creates the need to analyze the sociodemographic and psychosocial factors that are associated with these problems in a young population. It has been observed how sociodemographic and psychosocial variables also influence obesity $[6,7,9]$. On the other hand, there is controversy as to whether physical activity and energy expenditure can provide a solution 
to obesity; there are authors who are against their contribution [26-28] and in their favor [8,29-31]. In the present study, the authors considered as an object of interest the analysis of the Spanish population aged 18-30, as it is the first stage of adulthood and where it is believed that there is a great possibility of changing life habits [32,33]. Based on the aforementioned, the aim of the study was to know the influence and relationship of PA on BMI and sociodemographic and psychosocial characteristics in young Spanish adults aged 18-30 in the period of 2009-2017.

\section{Materials and Methods}

\subsection{Participants and Design}

A cross-sectional study was conducted in different years (2009, 2011/2012, 2014 and 2017) with different samples and registration of young adults aged 18-30 residing in Spain using records from the European Health Survey in Spain (EHSS) in 2009 [34] and 2014 [35] and the National Health Survey (NHS) in 2011/2012 [36] and 2017 [37]. The surveys were conducted by the National Statistics Institute (INE, abbreviation in Spanish) and the Ministry of Health, Social Services and Equality (MSSI, abbreviation in Spanish). In both surveys, personal interviews were conducted by the INE and MSSI using multi-stage probabilistic sampling by municipalities (first stage), sections (second stage) and individuals (third stage). Participants were selected by random sampling and fees based on sex and age. The inclusion criteria, for the data analyzed in the present study, were age 18-30 and a resident of Spain during the years of the surveys. The exclusion criteria were age under 18 or over 30 and inability to respond to the interview, whether due to disability, illness, ignorance of the language or any other circumstance. The data obtained from the surveys were extracted from web pages of the INE and MSSI in the form of anonymized microdata, thus no authorization was required for their use. According to Spanish law, no report from the ethics committee is required to use anonymous and public data from these institutions.

For the present study, all the records of young adults aged 18-30 were selected. The total sample of participants numbered 10,061: 2879 were from 2009, 2637 from 2011/2012, 2350 from 2014 and 2195 from 2017.

\subsection{Outcome Measures}

The dependent variable was the frequency of PA, which could be sedentary, perform physical activity occasionally, perform physical activity several times a month and perform physical activity several times a week.

The independent variables were: the year of the survey (quantitative variable), 2009, 2011/2012, 2014 or 2017; age (quantitative variable); sex (categorical), male or female; nationality (categorical), Spanish or foreigner; smoker (dichotomous), yes or no; marital status (categorical), single, married, divorced, widowed, separated and unifying separated, divorced or widowed in a single variable; educational level (categorical), without studies, primary, secondary, high school/vocational education and training or university; social class (established according to the categories proposed by the Spanish Society of Epidemiology [38]), which was stratified into the three levels of high class (Level I, directors and managers of companies with 10 or more employees and professionals with university degrees; and Level II, directors of companies with less than 10 employees and professionals with college diplomas), medium class (Level III, intermediate occupations, and Level IV, workers in qualified technical occupations), and low class (Level V, primary sector workers; and Level VI, unskilled workers); health self-perception (categorical), psychosocial problems measured by the Goldberg Health Questionnaire (GHQ12); depression (dichotomous) through direct questioning; and the BMI (quantitative variable), which was calculated from self-reported body weight and height. 


\subsection{Statistical Analysis}

The quantitative variables are expressed by means of the median and the mode, since they did not follow a normal distribution when performing the Kolmogorov-Smirnov normality test. Qualitative variables (categorical or dichotomous) are expressed by count $(n)$ and percentage (\%). The categorized variables were compared using chi-square tests for contingency tables; in the case of $2 \times 2$ tables, the chi-square statistic with Yates correction was used, and, when some expected frequency was $\leq 5$, Fisher's exact test was applied. Multiple logistic regression was performed to determine the influence of the variables on the frequency with which young adults performed PA. For this regression, it was established that they did not perform PA frequently if they described being sedentary or occasionally performing PA, while performing PA several times a month or several times a week established that they performed PA frequently. The Wald statistic was used, in which variables with $p \geq 0.15$ were eliminated one by one from the model. Odds ratios (ORs) were calculated with $95 \%$ confidence intervals. Statistical significance was considered with a value of $p<0.05$, and all hypothesis contrasts were bilateral. The statistical program IBM SPSS, version 24 (IBM Corp, Armonk, NY, USA), licensed for the University of Castilla-La Mancha (UCLM) was used for the statistical treatment of the data.

\section{Results}

The total sample of participants in the study numbered 10,061, with an age range of 18-30. The median age was 25 , and the mode age was 30 . In height, both the median and the mode were $170 \mathrm{~cm}$, and, in terms of weight, a median of $68 \mathrm{~kg}$ and a mode of $70 \mathrm{~kg}$ were obtained. Of the total sample, $48.6 \%$ were men versus $51.4 \%$ women, and it was observed that $81.6 \%$ were single, $17.3 \%$ married and $1.1 \%$ widowed, separated or divorced. Young adults who reported a state of health perceived as regular, bad or very bad accounted for $11.2 \%$ of the total sample. Of those surveyed, $8.7 \%$ reported a slight limitation and $1.3 \%$ a severe limitation in the last six months. In turn, 56.5\% of young adults reported that worry made them lose a lot of sleep.

Of the sample, $35.8 \%$ were smokers, of whom $53.6 \%$ were men, and a significant difference was found between sexes $(p<0.001)$. Being constantly overwhelmed and stressed was reported by $60.5 \%$ of the sample, of whom women represented $54.3 \%$, with a significant difference between sexes $(p<0.001)$. A feeling that they could not overcome their difficulties was reported by $48.9 \%$ of the total sample, which was composed of $47.4 \%$ men and $52.6 \%$ women $(p \geq 0.05)$. Of the total sample, $39.5 \%$ reported feeling unhappy, which was composed of $46.1 \%$ men and $53.9 \%$ women $(p<0.01)$. A loss of self-confidence was felt by $27.4 \%$ of the total sample, which was composed of $46.2 \%$ men and $53.8 \%$ women $(p<0.05)$. Table 1 shows the sociodemographic characteristics of the sample according to the years of the surveys.

Table 1. Sociodemographic characteristics of Spanish people 18-30 $(n=10,061)$ in the period 2009-2017.

\begin{tabular}{|c|c|c|c|c|c|}
\hline Characteristics & $\begin{array}{c}2009 \\
n=2879(\%)\end{array}$ & $\begin{array}{c}2011 / 2012 \\
n=2637(\%)\end{array}$ & $\begin{aligned} & 2014 \\
n= & 2350(\%)\end{aligned}$ & $\begin{aligned} & 2017 \\
n= & 2195(\%)\end{aligned}$ & $p$ \\
\hline $\begin{array}{l}\text { Age } \\
\text { Sex }\end{array}$ & 24.81 (SD 3.8) & 24.62(SD 3.76) & $24.68(\mathrm{SD} 3.76)$ & 24.37 (SD 3.82) & 0.15 \\
\hline Men & $1414(49.1)$ & $1290(48.9)$ & $1126(47.9)$ & $1056(48.6)$ & 0.787 \\
\hline $\begin{array}{l}\text { Women } \\
\text { Smoke }\end{array}$ & $1465(50.9)$ & 1347 (51.1) & $1224(52.1)$ & $1139(51.4)$ & 0.787 \\
\hline $\begin{array}{l}\text { Yes } \\
\text { No }\end{array}$ & $\begin{array}{l}1127(40.3) \\
1671(59.7)\end{array}$ & $\begin{array}{c}978(37.1) \\
1657(62.9)\end{array}$ & $\begin{array}{c}803(34.2) \\
1545(65.8)\end{array}$ & $\begin{array}{c}667(30.4) \\
1527(69.6)\end{array}$ & $<0.001$ \\
\hline $\begin{array}{l}\text { Nationality } \\
\text { Spanish } \\
\text { Foreigner }\end{array}$ & $\begin{array}{c}2478(86.1) \\
401(13.9)\end{array}$ & $\begin{array}{c}2298(87.1) \\
229(12.9)\end{array}$ & $\begin{array}{c}2056(87.5) \\
294(12.5)\end{array}$ & $\begin{array}{c}1931(88) \\
264(12)\end{array}$ & 0.211 \\
\hline $\begin{array}{c}\text { Marital status } \\
\text { Single } \\
\text { Married } \\
\text { Widowed }\end{array}$ & $\begin{array}{c}2298(79.7) \\
546(19) \\
2(0.1)\end{array}$ & $\begin{array}{c}2203(83.6) \\
405(15.4) \\
0(0)\end{array}$ & $\begin{array}{c}1896(80.7) \\
426(18.1) \\
2(0.1)\end{array}$ & $\begin{array}{c}1811(82.6) \\
361(16.4) \\
1(0)\end{array}$ & 0.014 \\
\hline
\end{tabular}


Table 1. Cont.

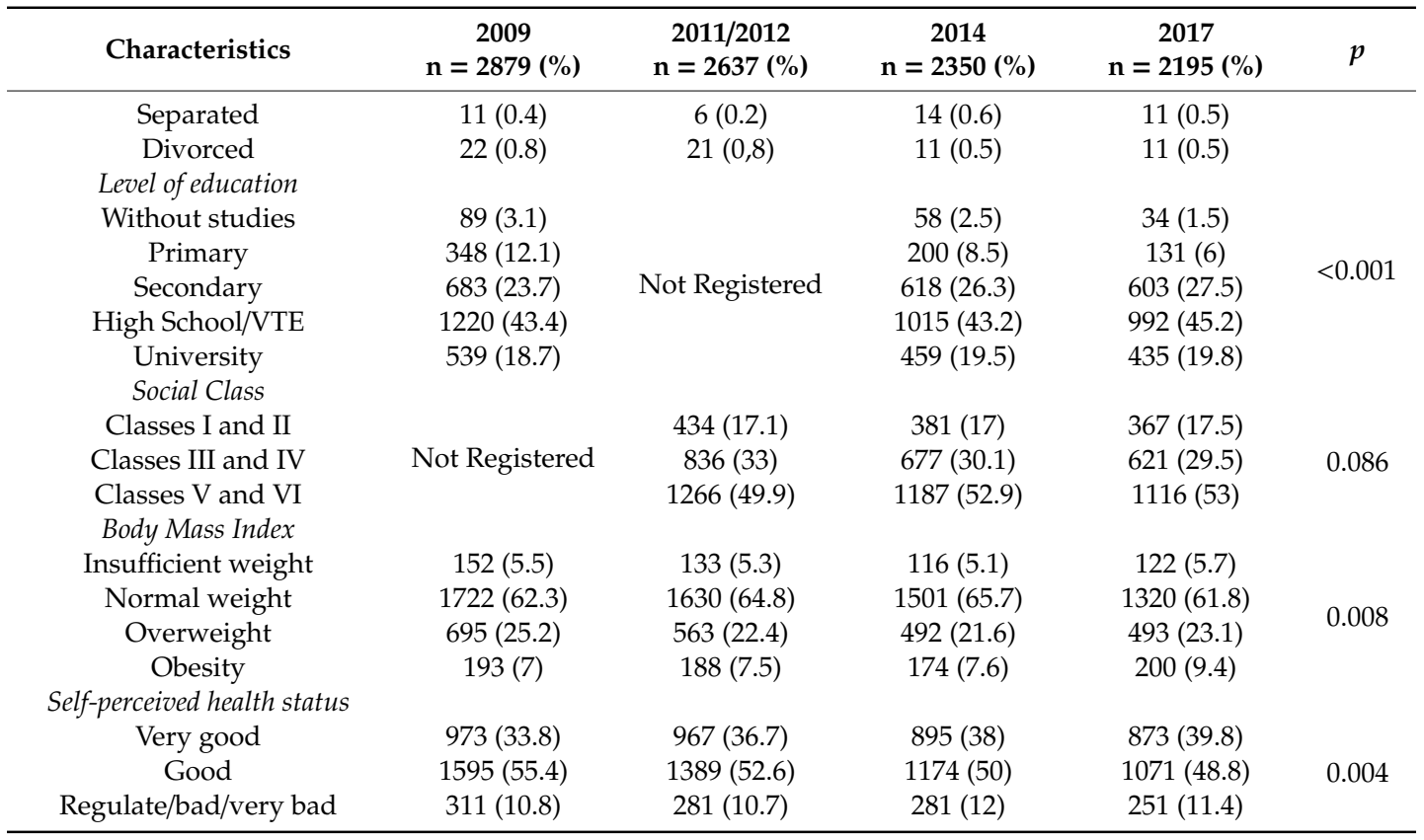

VTE, Vocational Education and Training.

Table 2 shows the anthropometric variables and their relationship with frequency of PA among the young adults studied. It can be seen that men had more PA than women $(p<0.001)$, that the percentage of smokers was lower among those who trained several times a week, and that those who have sedentary behavior are those who smoked the most. Regarding the percentage of people who did PA several times a week, it was observed that, in 2014, there was a higher percentage, with 2011/2012 being the year in which the fewest people did PA several times a week. Therefore, one could say that was the year in which a sedentary lifestyle was most common.

Table 2. Frequency of physical activity in relation to health self-perception and sociodemographic and psychosocial characteristics in young Spanish adults aged 18-30 (2009-2017).

\begin{tabular}{|c|c|c|c|c|c|}
\hline Characteristics & $\begin{array}{c}\text { Sedentary } \\
\mathrm{n}= \\
2464(34.4 \%)\end{array}$ & $\begin{array}{c}\text { Occasionally } \\
\text { PA } \\
n= \\
1933(26.9 \%)\end{array}$ & $\begin{array}{c}\text { PA Several } \\
\text { Times/Month } \\
n= \\
\mathbf{1 4 5 8 ( 2 0 . 3 \% )}\end{array}$ & $\begin{array}{c}\text { PA Several } \\
\text { Times/Week } \\
n= \\
1320(18.4 \%)\end{array}$ & $p$ \\
\hline \multicolumn{6}{|l|}{ Sex } \\
\hline Men & $883(35.8)$ & $760(39.3)$ & $969(66.5)$ & 857 (64.9) & \multirow{2}{*}{$<0.001$} \\
\hline Women & $1581(64.2)$ & $1173(60.7)$ & 489 (33.5) & $463(35.1)$ & \\
\hline \multicolumn{6}{|l|}{ Nationality } \\
\hline Spanish & $2066(83.8)$ & $1677(86.8)$ & 1325 (90.9) & $1212(91.8)$ & \multirow{2}{*}{$<0.001$} \\
\hline Foreigner & $398(16.2)$ & $256(13.2)$ & $133(9.1)$ & $108(8.2)$ & \\
\hline \multicolumn{6}{|l|}{ Self-perceived health status } \\
\hline Very good & $745(30.2)$ & $730(37.8)$ & $630(43.2)$ & $627(47.5)$ & \multirow{3}{*}{$<0.001$} \\
\hline Good & $1347(54.7)$ & $979(50.6)$ & $713(48.9)$ & $594(45)$ & \\
\hline Regulate/bad/very bad & $372(15.1)$ & $224(11.6)$ & $115(7.9)$ & $99(7.5)$ & \\
\hline \multicolumn{6}{|l|}{ Depression } \\
\hline Yes & $110(4.5)$ & $69(3.6)$ & $25(1.7)$ & $22(1.7)$ & \multirow{2}{*}{$<0.001$} \\
\hline No & $2354(95.5)$ & $1863(96.4)$ & $1432(98.3)$ & $1298(98.3)$ & \\
\hline \multicolumn{6}{|l|}{ Year Survey } \\
\hline $2011 / 2012$ & $1038(42.1)$ & $652(33.7)$ & $569(39)$ & $376(28.5)$ & \multirow{3}{*}{$<0.001$} \\
\hline 2014 & $698(28.3)$ & $687(35.5)$ & 477 (32.7) & $489(36.6)$ & \\
\hline 2017 & $728(29.5)$ & $594(30.7)$ & $412(28.3)$ & 461 (34.9) & \\
\hline \multicolumn{6}{|l|}{ Consumption of alcohol } \\
\hline Daily & $57(2.3)$ & $60(3.1)$ & $36(2.5)$ & $28(2.1)$ & \\
\hline 3-6 times/week & $88(3.6)$ & $86(4.5)$ & $80(5.5)$ & $53(4)$ & \\
\hline 1-2 times/week & $559(22.7)$ & $421(21.8)$ & $444(30.5)$ & $406(30.8)$ & $<0.001$ \\
\hline
\end{tabular}


Table 2. Cont.

\begin{tabular}{|c|c|c|c|c|c|}
\hline Characteristics & $\begin{array}{c}\text { Sedentary } \\
n= \\
2464(34.4 \%)\end{array}$ & $\begin{array}{c}\text { Occasionally } \\
\text { PA } \\
n= \\
\text { 1933(26.9\%) }\end{array}$ & $\begin{array}{c}\text { PA Several } \\
\text { Times/Month } \\
\text { n = } \\
\text { 1458(20.3\%) }\end{array}$ & $\begin{array}{c}\text { PA Several } \\
\text { Times/Week } \\
\text { n = } \\
\text { 1320(18.4\%) }\end{array}$ & $p$ \\
\hline 1-3 times/month & $948(38.5)$ & $831(43)$ & $634(43.5)$ & $598(45.3)$ & \\
\hline Teetotaler & $812(33)$ & $534(27.6)$ & $263(18.1)$ & $235(17.8)$ & \\
\hline \multicolumn{6}{|l|}{ Smoke } \\
\hline Yes & $984(39.9)$ & $656(34)$ & $463(31.8)$ & $344(26.1)$ & \multirow{2}{*}{$<0.001$} \\
\hline No & $1480(60.1)$ & $1274(66)$ & $994(68.2)$ & $976(73.9)$ & \\
\hline \multicolumn{6}{|l|}{ Ability to concentrate } \\
\hline Better than usual & $52(3)$ & $44(3.5)$ & $41(4.2)$ & $46(5.5)$ & \multirow{3}{*}{0.003} \\
\hline As usual & $1503(85.4)$ & $1076(86.6)$ & $844(86.1)$ & $725(86.8)$ & \\
\hline Worse than usual & 205 (11.6) & $122(9.8)$ & $95(9.7)$ & $64(7.7)$ & \\
\hline \multicolumn{6}{|l|}{ Loss of sleep due to worries } \\
\hline No, absolutely & $721(41)$ & $502(40.4)$ & $428(43.7)$ & $445(53.4)$ & \multirow{3}{*}{$<0.001$} \\
\hline No more than normal & $679(38.6)$ & $483(38.9)$ & $396(40.4)$ & $247(29.6)$ & \\
\hline $\begin{array}{l}\text { More than normal } \\
\text { Ability to decide }\end{array}$ & $360(20.5)$ & $257(20.7)$ & $156(15.9)$ & $142(17)$ & \\
\hline More than usual & $126(7.2)$ & $109(8.8)$ & $84(8.6)$ & $89(10.7)$ & \multirow{3}{*}{$<0.001$} \\
\hline As usual & $1541(87.6)$ & 1095 (88.2) & $859(87.7)$ & $727(87.1)$ & \\
\hline Less than usual & $93(5.3)$ & $38(3.1)$ & $36(3.7)$ & $19(2.3)$ & \\
\hline \multicolumn{6}{|l|}{ Ability to face problems } \\
\hline More than usual & $96(5.5)$ & $77(6.2)$ & $46(4.7)$ & $67(8)$ & \multirow{3}{*}{$<0.001$} \\
\hline As usual & $1560(88.7)$ & $1106(89)$ & $896(91.5)$ & $754(90.4)$ & \\
\hline Less than usual & $103(5.9)$ & $59(4.8)$ & $37(3.8)$ & $13(1.6)$ & \\
\hline \multicolumn{6}{|l|}{ Loss of self-confidence } \\
\hline No, absolutely & $1225(69.6)$ & 908 (73.1) & 707 (72.1) & $658(78.8)$ & \multirow{2}{*}{$<0.001$} \\
\hline $\begin{array}{l}\text { Equal o more than normal } \\
\text { Feeling happy }\end{array}$ & $536(30.4)$ & $334(26.9)$ & $273(27.9)$ & $177(21.2)$ & \\
\hline More than usual & $153(8.7)$ & $123(9.9)$ & $79(8.1)$ & $112(13.4)$ & \multirow{3}{*}{$<0.001$} \\
\hline As usual & $1494(84.8)$ & $1062(85.5)$ & $871(89.1)$ & $691(82.8)$ & \\
\hline Less than usual & $114(6.5)$ & $57(4.6)$ & $28(2.9)$ & $32(3.8)$ & \\
\hline
\end{tabular}

The BMI data in the different years of the surveys can be seen in Figures 1-3. The BMI of the total sample can be observed in Figure $1(p<0.01)$.

The BMI of men is presented in Figure $2(p>0.05)$, which shows how the percentage of overweight men decreased from a value of $32 \%$ in 2009 to $20.2 \%$ in 2017. The BMI of women is shown in Figure 3 $(p<0.05)$, which shows the percentage of obese women rose from $23.2 \%$ in 2009 to $27.2 \%$ in 2017 . BMI and its relation to educational level can be seen in Figure $4(p<0.001)$, where $69.7 \%$ of people with university studies had a normal weight as compared to $48.4 \%$ of people without studies.

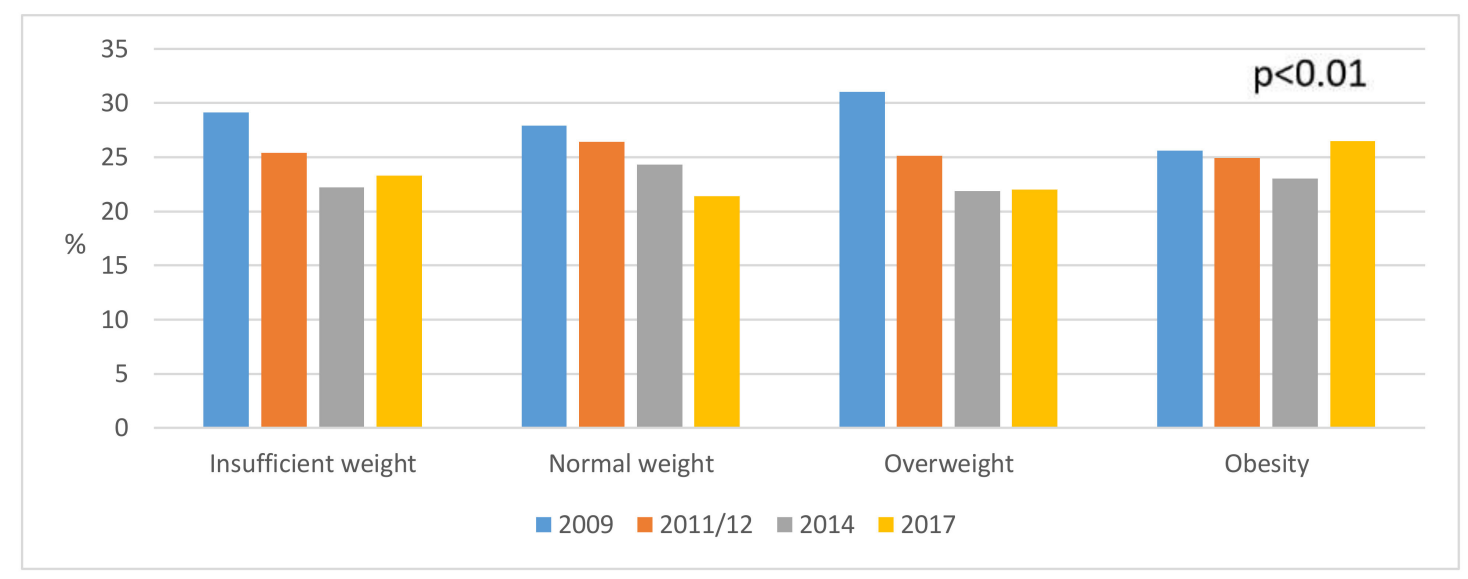

Figure 1. Weight status in the total simple $(n=10,061)(2009-2017)$. 


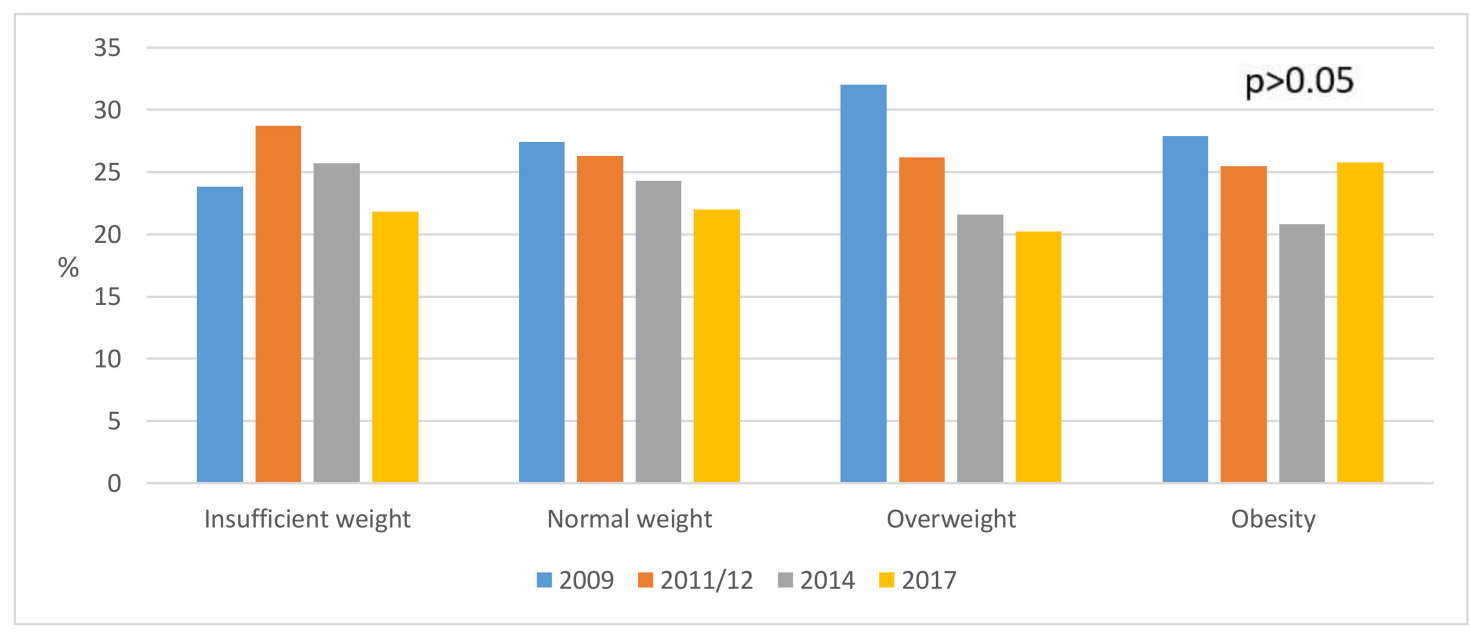

Figure 2. Weight status in young male adults $(n=4886)(2009-2017)$.

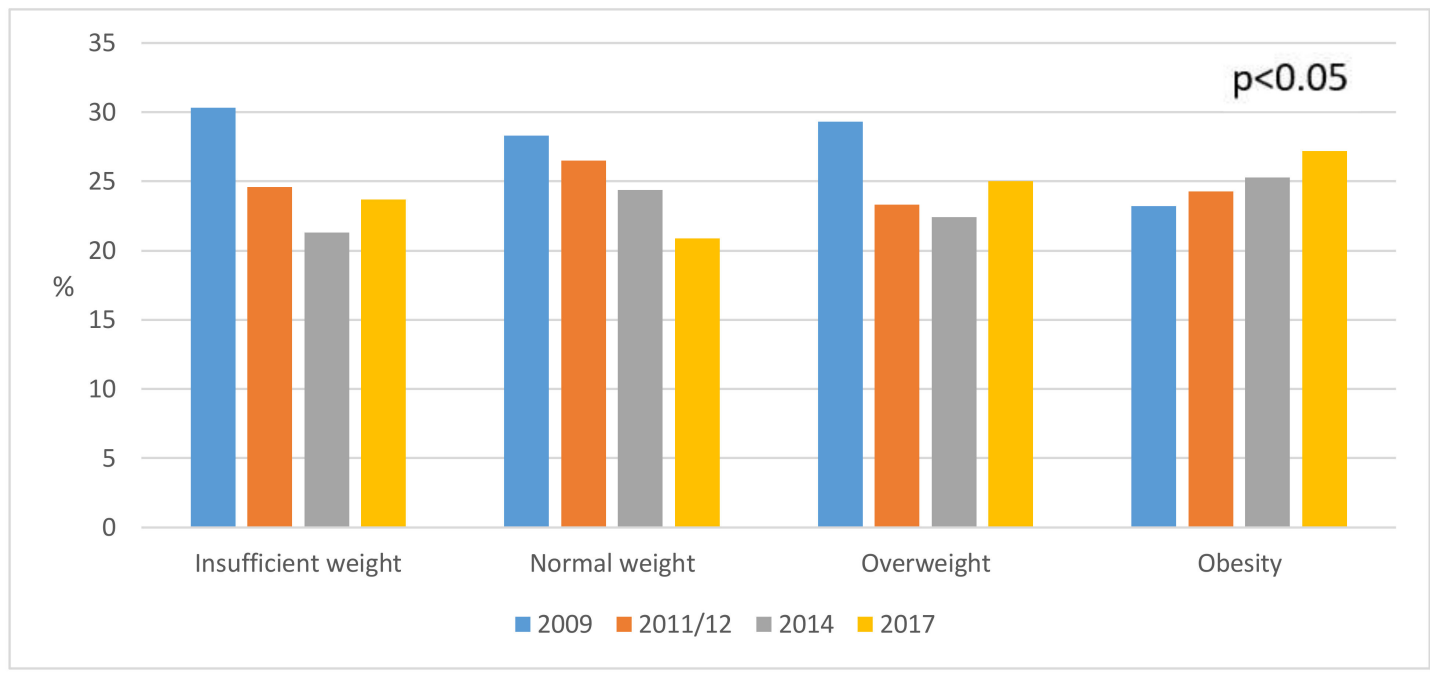

Figure 3. Weight status in young female adults $(n=5175)(2009-2017)$.

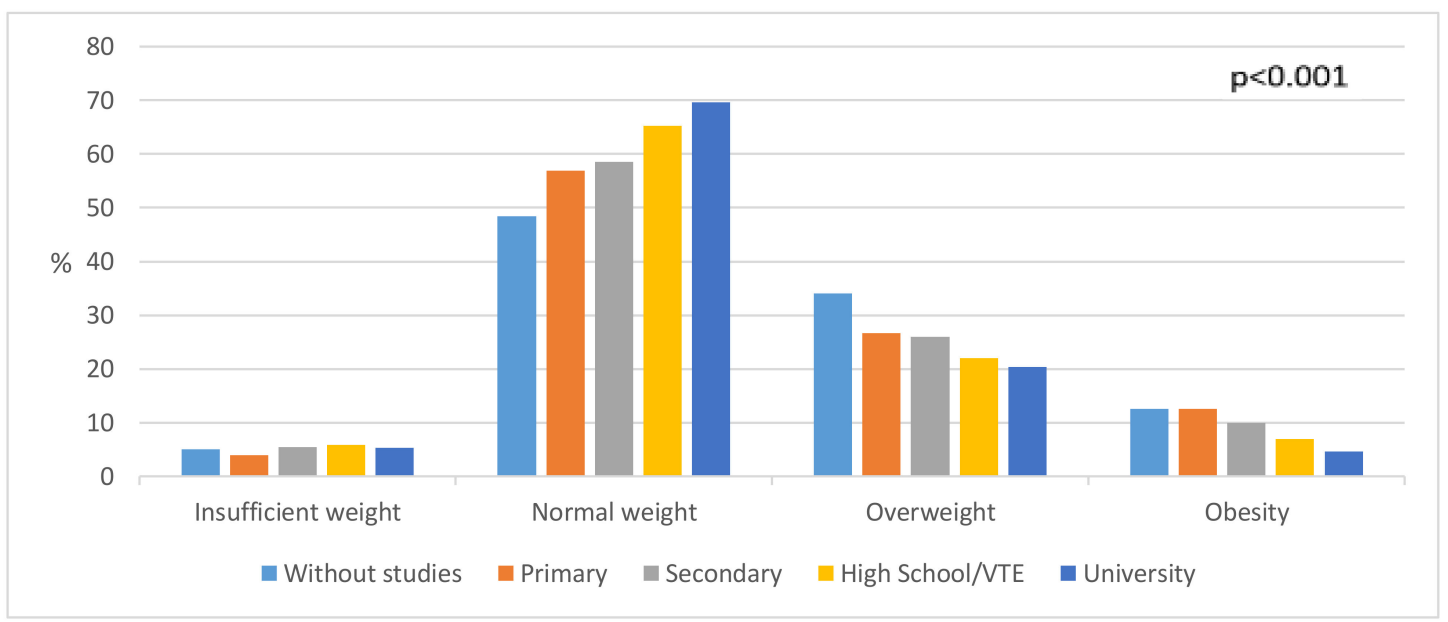

Figure 4. Distribution of weight status at the educational level (2009-2017). VTE, Vocational Education and Training.

Of the total sample, 5.4\% were underweight, $63.7 \%$ were of normal weight and $30.9 \%$ overweight or obese. Looking at the distribution by weight, $8 \%$ of the participants in the surveys weighed less than 
$50 \mathrm{~kg}, 51.8 \%$ weighed $51-70 \mathrm{~kg}, 33.1 \%$ weighed $71-90 \mathrm{~kg}, 5.9 \%$ weighed $91-110 \mathrm{~kg}$ and $1.2 \%$ weighed more than $110 \mathrm{~kg}$. Table 3 shows the relationship between BMI and different anthropometric variables in the sample.

Table 3. Body mass index related to sociodemographic and psychosocial variables, health status, alcohol consumption and frequency of physical activity in young Spanish adults aged 18-30 (2009-2017).

\begin{tabular}{|c|c|c|c|c|c|}
\hline Characteristics & $\begin{array}{c}\text { Insufficient } \\
\text { Weight } \\
\mathrm{n}=523(5.4 \%)\end{array}$ & $\begin{array}{l}\text { Normal Weight } \\
n=6173(63.7 \%)\end{array}$ & $\begin{array}{c}\text { Overweight } \\
\mathrm{n}=2243(23.1 \%)\end{array}$ & $\begin{array}{c}\text { Obesity } \\
\mathrm{n}=755(7.8 \%)\end{array}$ & $p$ \\
\hline \multicolumn{6}{|l|}{ Sex } \\
\hline Men & $101(19.3)$ & $2831(45.9)$ & $1414(63)$ & $384(50.9)$ & \multirow{2}{*}{$<0.001$} \\
\hline Women & $422(80.7)$ & $3342(54.1)$ & $829(37)$ & $371(49.1)$ & \\
\hline \multicolumn{6}{|l|}{ Nationality } \\
\hline Spanish & $469(89.7)$ & $5425(87.9)$ & $1910(85.2)$ & $658(87.2)$ & \multirow{3}{*}{0.003} \\
\hline Foreigner & $54(10.3)$ & $748(12.1)$ & $333(14.8)$ & $97(12.8)$ & \\
\hline \multicolumn{5}{|l|}{ Age } & \\
\hline $18-22$ & $263(50.3)$ & $2174(35.2)$ & $527(23.5)$ & $165(21.9)$ & \multirow{3}{*}{$<0.001$} \\
\hline $23-26$ & $145(27.7)$ & $1864(30.2)$ & $629(28)$ & $201(27.8)$ & \\
\hline $27-30$ & $115(22)$ & $2135(34.6)$ & $1087(48.5)$ & $380(50.3)$ & \\
\hline \multicolumn{6}{|l|}{ Marital status } \\
\hline Single & $470(89.9$ & $5204(84.3)$ & $1704(76)$ & $539(71.4)$ & \multirow{3}{*}{$<0.001$} \\
\hline Married & $49(9.4)$ & $894(14.5)$ & $513(22.9)$ & $212(28.1)$ & \\
\hline $\begin{array}{l}\text { Wid/Sepa/Divor } \\
\text { Social Class }\end{array}$ & \multicolumn{4}{|c|}{ Social Class } & \\
\hline Class I and II & $78(22.4)$ & $811(19)$ & $218(14.5)$ & $48(9)$ & \multirow{3}{*}{$<0.001$} \\
\hline Class III and IV & $118(33.9)$ & $1360(32)$ & $435(29)$ & $165(30.8)$ & \\
\hline Class V and VI & $152(43.7)$ & $2090(49)$ & $847(56.5)$ & $323(51.3)$ & \\
\hline \multicolumn{6}{|l|}{ Self-perceived health status } \\
\hline Very good & $178(34)$ & $2418(39.3)$ & $773(34.5)$ & $197(26.1)$ & \multirow{3}{*}{$<0.001$} \\
\hline Good & $274(52.4)$ & $3135(50.8)$ & $1208(53.9)$ & $428(56.7)$ & \\
\hline Regulate/bad/very bad & $71(13.6)$ & $610(9.9)$ & $262(11.6)$ & $130(17.2)$ & \\
\hline \multicolumn{6}{|l|}{ Depression } \\
\hline Yes & $17(3.3)$ & $155(2.5)$ & $73(3.3)$ & $44(5.8)$ & \multirow[b]{2}{*}{$<0.001$} \\
\hline No & $506(96.7)$ & 6017 (97.5) & $2170(96.7)$ & $711(94.2)$ & \\
\hline \multicolumn{6}{|l|}{ PA frequency } \\
\hline Sedentary & $156(42)$ & $1377(31)$ & $538(34.9)$ & $269(47.9)$ & \multirow{4}{*}{$<0.001$} \\
\hline Occasionally PA & $92(24.8)$ & $1211(27.2)$ & $387(25)$ & $170(30.2)$ & \\
\hline PA several times/month & $70(18.9)$ & $952(21.4)$ & 339 (21.9) & $66(11.7)$ & \\
\hline PA several times/week & $53(14.3)$ & $907(20.4)$ & $282(18.2)$ & $57(10.1)$ & \\
\hline \multicolumn{6}{|l|}{ Consumption of alcohol } \\
\hline Daily & $11(2.1)$ & $139(2.3)$ & $75(3.4)$ & $13(1.7)$ & \multirow{4}{*}{$<0.001$} \\
\hline 3-6 times/week & $17(3.3)$ & $266(4.3)$ & $109(4.9)$ & $15(2)$ & \\
\hline 1-2 times/week & 91 (17.6) & $1364(22.3)$ & $521(23.5)$ & $121(16.2)$ & \\
\hline $1-3$ times/month & $262(50.8)$ & $2893(47.2)$ & $947(42.7)$ & $330(44.2)$ & \\
\hline Teetotaler & $135(26.2)$ & $1468(2 \mathrm{I})$ & $566(25.5)$ & $268(35.9)$ & \\
\hline \multicolumn{6}{|l|}{ Anxiety } \\
\hline Yes & $29(5.5)$ & $249(4)$ & $100(4.5)$ & $55(7.3)$ & \multirow{2}{*}{$<0.001$} \\
\hline No & $494(94.5)$ & $5921(96)$ & $2142(95.5)$ & $700(92.7)$ & \\
\hline Weight & & & & & \\
\hline$<50 \mathrm{~kg}$ & $332(63.5)$ & $430(7)$ & $1(0)$ & $0(0)$ & \\
\hline $51-70 \mathrm{~kg}$ & $191(36.5)$ & 4363 (70.7) & $468(20.9)$ & $4(0.5)$ & \\
\hline $71-90 \mathrm{~kg}$ & $0(0)$ & $1367(22.1)$ & $1530(68.2)$ & $315(41.7)$ & $<0.001$ \\
\hline $91-110 \mathrm{~kg}$ & $0(0)$ & $13(0.2)$ & $242(10.8)$ & $322(42.6)$ & \\
\hline$>110 \mathrm{~kg}$ & $0(0)$ & $0(0)$ & $2(0.1)$ & $114(15.1)$ & \\
\hline Feel that you are worth nothing & & & & & \\
\hline No, absolutely & $200(78.7)$ & $2418(82.2)$ & $834(79.2)$ & $300(77.3)$ & \\
\hline No more than normal & 46 (18.1) & 457 (15.5) & $185(17.6)$ & $64(16.5)$ & 0.001 \\
\hline More than normal & $8(3.1)$ & $68(2.3)$ & $34(3.2)$ & $24(6.8)$ & \\
\hline Feeling unhappy & & & & & \\
\hline No, absolutely & $132(52)$ & $1829(62.1)$ & $638(60.6)$ & $210(54.1)$ & \\
\hline Equal o more than normal & $122(48)$ & $1116(37.9)$ & $415(39.4)$ & $178(45.9)$ & 0.001 \\
\hline
\end{tabular}

Wid, widowed; Sepa, Separated; Divor, Divorced; PA, physical activity.

The logistic regression analysis presented in Table 4 shows the frequency of PA and its association with normal weight (OR 1.66, 95\% CI 1.25-2.20, $p=0.001)$, alcohol consumption (1-2 times/week 
(OR 1.97, 95\% CI 1.47-2.63, $p<0.001$ ) and 1-3 times/month (OR 1.56, 95 CI 1.22-2, $p<0.001)$ ), being male (OR 2.63, 95\% CI 2.09-3.32, $p<0.001$ ), being single (OR 2.11, 95\% CI 1.58-2.8, $p<0.001$ ), university studies (OR 2.66, 95\% CI 0.99-7.18, $p=0.054$ ), feeling more happy than usual (OR 1.97, 95\% CI 1.09-3.58, $p=0.026$ ), feeling happy as usual (OR 1.55, 95\% CI 0.91-2.66, $p=0.110$ ), Social Classes I and II (OR 1.34, 95\% CI 1.02-1.78, $p=0.042$ ), Social Classes II and IV (OR 1.35, 95\% CI 1.08-1.70, $p=0.008$ ), smoking (OR 1.66, 95\% CI 1.33-2.08, $p<0.001$ ) and weighing 71-90 kg (OR 1.98, 95\% CI 0.83-4.70, $p=0.122$ ).

Table 4. Logistic regression model for the association between the frequency of physical activity, BMI and sociodemographic and psychosocial characteristics of young Spanish adults aged 18-30 (2009-2017).

\begin{tabular}{|c|c|c|}
\hline & OR $(95 \% \mathrm{CI})$ & $p$ \\
\hline \multicolumn{3}{|l|}{ Body Mass Index } \\
\hline Insufficient weight & $1.24(0.71-2.17)$ & 0.458 \\
\hline Normal weight & $1.66(1.25-2.20)$ & 0.001 \\
\hline Overweight/Obesity & Reference & \\
\hline \multicolumn{3}{|l|}{ Consumption of alcohol } \\
\hline Daily & $1.21(0.62-2.35)$ & 0.577 \\
\hline 3-6 times/week & $0.966(0.59-1.58)$ & 0.890 \\
\hline $1-2$ times/week & $1.97(1.47-2.63)$ & $<0.001$ \\
\hline 1-3 times/month & $1.56(1.22-2)$ & $<0.001$ \\
\hline Teetotaler & Reference & \\
\hline \multicolumn{3}{|l|}{ Sex } \\
\hline Male & $2.63(2.09-3.32)$ & $<0.001$ \\
\hline Female & Reference & \\
\hline \multicolumn{3}{|l|}{ Marital status } \\
\hline Single & $2.11(1.58-2.8)$ & $<0.001$ \\
\hline $\begin{array}{c}\text { Married/Widowed/Separated/Divorced } \\
\text { Level of education }\end{array}$ & Reference & \\
\hline Without education & Reference & \\
\hline Primary & $1.04(0.36-3.00$ & 0.944 \\
\hline Secondary & $1.59(0.60-4.23)$ & 0.351 \\
\hline High School/VTE & $1.98(0.75-5.23)$ & 0.169 \\
\hline University & $2.66(0.99-7.18)$ & 0.054 \\
\hline \multicolumn{3}{|l|}{ Feeling happy } \\
\hline More than usual & $1.97(1.09-3.58)$ & 0.026 \\
\hline As usual & $1.55(0.91-2.66)$ & 0.110 \\
\hline & Social class & \\
\hline Classes I and II & $1.34(1.02-1.78)$ & 0.042 \\
\hline Classes III and IV & $1.35(1.08-1.70)$ & 0.008 \\
\hline Classes V and VI & Reference & \\
\hline \multicolumn{3}{|l|}{ Smoke } \\
\hline Yes & Reference & \\
\hline No & $1.66(1.33-2.08)$ & $<0.001$ \\
\hline \multicolumn{3}{|l|}{ Weight } \\
\hline$<50 \mathrm{~kg}$ & $1.11(0.41-2.99)$ & 0.844 \\
\hline $51-70 \mathrm{~kg}$ & $1.18(0.48-2.91)$ & 0.717 \\
\hline $71-90 \mathrm{~kg}$ & $1.98(0.83-4.70)$ & 0.122 \\
\hline $91-110 \mathrm{~kg}$ & $1.60(0.65-3.99)$ & 0.310 \\
\hline$>110 \mathrm{~kg}$ & Reference & \\
\hline
\end{tabular}

\section{Discussion}

The aim of the study was to know the influence and relationship of PA on BMI and sociodemographic and psychosocial characteristics in young Spanish adults aged 18-30 during 2009-2017. The study found that obesity increased according to the age of the respondents, rising from 
$21.9 \%$ between 18 and 22 years old to $50.3 \%$ between 27 and 30 years old. Another finding was that a sedentary lifestyle decreased considerably from $42.1 \%$ in $2011 / 2012$ to $29.5 \%$ in 2017 .

A high BMI and depression are two current problems in society carrying a significant burden in terms of disease, functional disability and mortality $[3,19,20,22,39,40]$. In line with our findings, it has been found that obesity increases the risk of depression [41,42] and that the reverse is also true [43], with there being a bidirectional relationship. Physical activity seems to have a significant relationship with depression $[39,44,45]$ and with overweight and obesity $[5,46]$. A possible solution to improve both pathologies is PA. This study shows that obese people have more than twice as much depression as those with normal weight. In sedentary youth, there is the highest rate of obesity and depression. Trends have been observed that depressed obese people do less PA than obese people without depression [39].

In the different years of the study, sedentary lifestyle significantly reduced and frequency of PA increased significantly to several times a week among young adults between 2011/2012 and 2017. However, there was also an increase in obesity between 2011/2012 and 2017; these results are similar to other studies $[47,48]$. This could be due to the fact that obesity in the total sample is influenced by obesity among women, which has increased progressively in every year of the survey. In men, obesity decreased in 2014 (the year in which obesity fell in the total sample), when PA increased to several times per week, and they had a less sedentary lifestyle; 2011/2012 and 2017 showed similar values. The incidence of obesity was stable in men and increased in women, which may be due to men doing 2.63 times more PA than women and is similar to data in another recent study [48].

Among the results presented, it is stated that PA in the young adults surveyed is related to levels of happiness, although other studies refer to the relationship between PA and quality of life, self-esteem and affection [9,49]. With regard to happiness, failure to maintain adequate PA is associated with lower levels of mental health, including depression [50]. In our study, sedentary lifestyle and obesity are associated with a higher percentage of respondents who reported having suffered depression, a finding that is also corroborated in the literature $[39,44,45,51]$.

The differences in relation to social class are also reflected in the figures of obesity and overweight in Social Classes I and II and in Social Classes V and VI, with the values of Social Classes V and VI (5.7 and 3.9, respectively) greater than Social Classes I and II. In this case, it should be said that Social Classes V and VI showed the highest percentage of respondents. There are studies that show results similar to those found in this work, reflecting the relationship of obesity with social class and parents' BMI status [52-54]. In addition, it can be seen how obesity is related to PA, since Social Classes I and II and Social Classes III and IV have ORs of 1.34 and 1.35, respectively, in relation to doing more PA than Social Classes V and VI. A similar situation occurs with the educational level of the respondents, in which it is seen that those who have university studies do 2.66 times more PA than respondents without studies. Similarly, a higher educational level correlates with lower obesity, lower overweight, lower underweight and higher normal weight, similar results to those obtained by Peterson [55].

Alcohol consumption among young adults is related to BMI. It is observed that young adults with obesity have a higher rate of alcohol consumption as compared to those with normal weight, which is similar to the study by Monteiro et al. [56]. In relation to alcohol consumption and the frequency of PA of young adults, it is observed that those who do PA consume more alcohol than those who do not, coinciding with the findings of a recent review [57].

The finding of a relationship between a healthy lifestyle including PA and an unhealthy lifestyle including alcohol consumption may be surprising, and it may be due to a more active social life, as argued by other authors [54,58]. However, we observe in this work how, with tobacco, we obtain opposite values, since sedentary young adults are 1.66 times more likely to smoke than those who do PA.

Physical activity programs, including programs involving parents in healthy habits for their children [59], the use of mobile PA applications [60], recreational activities that require movement [61] and virtual reality implementation [62], among others, are interventions that offer positive results in increasing levels of PA and reducing obesity in the young population. 


\section{Limitations}

A limitation of this study is that PA was not objectively quantified with accelerometers or similar devices, as it was collected through self-reported questionnaires. With more objective instruments, the data collected would have greater validity and could avoid a possible altered perception by respondents. Another limitation is that dietary control data were not available for association with BMI, which was calculated by weight and self-referenced height; therefore, it was not measured with sensitivity devices indicating body composition, such as the fat mass and muscle mass of participants, which would have provided more detailed information on participants' body composition and the association with PA. With a more precise control of the diet and evaluation of body composition, it could be known if the increase in weight produced in 2017 was due to fat mass or muscle mass. Therefore, it would be interesting to carry out future studies that took these characteristics into account.

However, among the strengths of the study is the sample size, which is nationally representative and updated evidence on the situation of young adults in Spain, in relation to obesity and PA, as well as the circumstances that concur, among which that PA does help reduce obesity. All of this will allow for coherent intervention patterns.

\section{Conclusions}

In the period between 2011/2012 and 2017, sedentary lifestyle in young adults was reduced and PA was increased, with men being more active than women.

In this study, the realization of PA several times a week was associated with being a man, being a non-smoker, having a very good perception of one's health, having good mental health and being happy and self-confident.

Obesity was associated with young adults between 27 and 30 years old, who belong to Social Class V or VI, lead a sedentary lifestyle and are without studies. Over the study period, obesity was increased in women and decreased in men.

As implications for practice, the data indicate the need to propose programs that promote PA and diet control in young adults, aiming at a higher uptake among young women to curb the increase in overweight and obesity levels in this population in order to try to reverse them in later adulthood and achieve habits to maintain a healthy state throughout life.

Author Contributions: Conceptualization, J.A.L.-A. and J.M.C.-T.; methodology, J.A.L.-A. and M.M.M.; software, J.A.L-A.; validation, A.I.C.-C., E.S.-S., M.M.M., M.A.R.-B. and P.J.L.-S.; formal analysis, J.A.L.-A. and J.M.C.-T.; investigation, J.A.L.-A., E.S.-S., M.A.R.-B. and A.I.C.-C.; resources, J.A.L.-A., P.J.L-S., E.S.-S. and A.I.C.-C.; data curation, J.A.L.-A. and M.A.R.-B.; writing-original draft preparation, J.A.L.-A. and J.M.C.-T.; writing-review and editing, J.A.L.-A., A.I.C.-C., E.S.-S., M.M.M., M.A.R.-B., P.J.L.-S. and J.M.C.-T.; visualization, J.A.L.-A.; supervision, J.A.L.-A., A.I.C.-C., E.S.-S., M.M.M., M.A.R.-B., P.J.L.-S. and J.M.C.-T.; project administration, J.A.L.-A.; and funding acquisition, J.A.L.-A., A.I.C.-C., E.S.-S., M.M.M., M.A.R.-B., P.J.L.-S. and J.M.C.-T.

Funding: This research was funded by a grant from the European Regional Development Fund (ERDF) [Fondo Europeo de Desarrollo Regional (FEDER) (DOCM 24/01/19)].

Conflicts of Interest: The authors declare no conflict of interest.

\section{References}

1. Carson, V.; Hunter, S.; Kuzik, N.; Wiebe, S.A.; Spence, J.C.; Friedman, A.; Tremblay, M.S.; Slater, L.; Hinkley, T. Systematic review of physical activity and cognitive development in early childhood. J. Sci. Med. Sport 2016, 19, 573-578. [CrossRef] [PubMed]

2. Simmonds, M.; Llewellyn, A.; Owen, C.G.; Woolacott, N. Predicting adult obesity from childhood obesity: A systematic review and meta-analysis. Obes. Rev. 2016, 17, 95-107. [CrossRef] [PubMed]

3. Staiano, A.E.; Marker, A.M.; Martin, C.K.; Katzmarzyk, P.T. Physical activity, mental health, and weight gain in a longitudinal observational cohort of nonobese young adults. Obesity 2016, 24, 1969-1975. [CrossRef] [PubMed] 
4. INE. Población Residente Por Fecha, SExo Y Edad. 2018. Available online: http://www.ine.es (accessed on 21 December 2018).

5. Drenowatz, C.; Gribben, N.; Wirth, M.D.; Hand, G.A.; Shook, R.P.; Burgess, S.; Blair, S.N. The Association of Physical Activity during Weekdays and Weekend with Body Composition in Young Adults. J. Obes. 2016, 2016, 1-8. [CrossRef]

6. Archer, E.; Lavie, C.J.; McDonald, S.M.; Thomas, D.M.; Hébert, J.R.; Ross, S.E.; McIver, K.L.; Malina, R.M.; Blair, S.N. Maternal Inactivity: 45-Year Trends in Mothers' Use of Time. Mayo Clin. Proc. 2013, 88, 1368-1377. [CrossRef]

7. McCormack, G.R.; Virk, J.S. Driving towards obesity: A systematized literature review on the association between motor vehicle travel time and distance and weight status in adults. Prev. Med. Baltim. 2014, 66, 49-55. [CrossRef]

8. Shook, R.P.; Hand, G.A.; Drenowatz, C.; Hebert, J.R.; Paluch, A.E.; Blundell, J.E.; Hill, J.O.; Katzmarzyk, P.T.; Church, T.S.; Blair, S.N. Low levels of physical activity are associated with dysregulation of energy intake and fat mass gain over 1 year. Am. J. Clin. Nutr. 2015, 102, 1332-1338. [CrossRef]

9. Leskinen, T.; Kujala, U.M. Health-Related Findings among Twin Pairs Discordant for Leisure-Time Physical Activity for 32 Years: The TWINACTIVE Study Synopsis. Twin Res. Hum. Genet. 2015, 18, $266-272$. [CrossRef]

10. Arem, H.; Moore, S.C.; Patel, A.; Hartge, P.; Berrington de Gonzalez, A.; Visvanathan, K.; Campbell, P.T.; Freedman, M.; Weiderpass, E.; Adami, H.-O.; et al. Leisure Time Physical Activity and Mortality. JAMA Intern. Med. 2015, 175, 959. [CrossRef]

11. Eijsvogels, T.M.H.; Thompson, P.D. Exercise Is Medicine: At any dose? JAMA 2015, 314, 1915. [CrossRef]

12. Schnohr, P.; O'Keefe, J.H.; Marott, J.L.; Lange, P.; Jensen, G.B. Dose of jogging and long-term mortality: The Copenhagen City Heart Study. J. Am. Coll. Cardiol. 2015, 65, 411-419. [CrossRef] [PubMed]

13. Laddu, D.R.; Rana, J.S.; Murillo, R.; Sorel, M.E.; Quesenberry, C.P., Jr.; Allen, N.B.; Gabriel, K.P.; Carnethon, M.R.; Liu, K.; Reis, J.P.; et al. 25-Year Physical Activity Trajectories and Development of Subclinical Coronary Artery Disease as Measured by Coronary Artery Calcium: The Coronary Artery Risk Development in Young Adults (CARDIA) Study. Mayo Clin. Proc. 2017, 92, 1660-1670. [CrossRef] [PubMed]

14. Haskell, W.L.; Lee, I.M.; Pate, R.R.; Powell, K.E.; Blair, S.N.; Franklin, B.A.; Macera, C.A.; Heath, G.W.; Thompson, P.D.; Bauman, A. Physical Activity and Public Health: Updated Recommendation for Adults from the American College of Sports Medicine and the American Heart Association. Circulation 2007, 116, 1081. [CrossRef] [PubMed]

15. Andersson, C.; Lyass, A.; Larson, M.G.; Spartano, N.L.; Vita, J.A.; Benjamin, E.J.; Murabito, J.M.; Esliger, D.W.; Blease, S.J.; Hamburg, N.M.; et al. Physical Activity Measured by Accelerometry and its Associations with Cardiac Structure and Vascular Function in Young and Middle-Aged Adults. J. Am. Heart Assoc. 2015, 4, e001528. [CrossRef] [PubMed]

16. Katzmarzyk, P.T.; Lee, I.-M. Sedentary behaviour and life expectancy in the USA: A cause-deleted life table analysis. BMJ Open 2012, 2, e000828. [CrossRef]

17. Shah, R.V.; Murthy, V.L.; Colangelo, L.A.; Reis, J.; Venkatesh, B.A.; Sharma, R.; Abbasi, S.A.; Goff, D.C.; Carr, J.J.; Rana, J.S.; et al. Association of Fitness in Young Adulthood with Survival and Cardiovascular Risk. JAMA Intern. Med. 2016, 176, 87-95. [CrossRef]

18. Laredo-Aguilera, J.A.; Carmona-Torres, J.M.; García-Pinillos, F.; Latorre-Román, P.Á. Effects of a 10-week functional training programme on pain, mood state, depression, and sleep in healthy older adults. Psychogeriatrics 2018, 18, 292-298. [CrossRef]

19. Gregor, M.F.; Hotamisligil, G.S. Inflammatory Mechanisms in Obesity. Annu. Rev. Immunol. 2011, 29 , 415-445. [CrossRef]

20. Ng, M.; Fleming, T.; Robinson, M.; Thomson, B.; Graetz, N.; Margono, C.; Mullany, E.C.; Biryukov, S.; Abbafati, C.; Abera, S.F.; et al. Global, regional, and national prevalence of overweight and obesity in children and adults during 1980-2013: A systematic analysis for the Global Burden of Disease Study 2013. Lancet 2014, 84, 766-781. [CrossRef]

21. WHO. Informe de la Comisión Para Acabar Con la Obesidad Infantil. World Health Organization. 2016. Available online: http:/www.who.int/end-childhood-obesity/publications/echo-report/es/ (accessed on 22 December 2018). 
22. Gortmaker, S.L.; Swinburn, B.A.; Levy, D.; Carter, R.; Mabry, P.L.; Finegood, D.T.; Huang, T.; Marsh, T.; Moodie, M.L. Changing the future of obesity: Science, policy, and action. Lancet 2011, 378, 838-847. [CrossRef]

23. Kakinami, L.; O’Loughlin, E.K.; Brunet, J.; Dugas, E.N.; Constantin, E.; Sabiston, C.M.; O'Loughlin, J. Associations between physical activity and sedentary behavior with sleep quality and quantity in young adults. Sleep Health 2017, 3, 56-61. [CrossRef] [PubMed]

24. Wu, J.; Wu, H.; Wang, J.; Guo, L.; Deng, X.; Lu, C. Associations between Sleep Duration and Overweight/Obesity: Results from 66,817 Chinese Adolescents. Sci. Rep. 2015, 5, 16686. [CrossRef] [PubMed]

25. Plotnikoff, R.C.; Costigan, S.A.; Williams, R.L.; Hutchesson, M.J.; Kennedy, S.G.; Robards, S.L.; Allen, J.; Collins, C.E.; Callister, R.; Germov, J. Effectiveness of interventions targeting physical activity, nutrition and healthy weight for university and college students: A systematic review and meta-analysis. Int. J. Behav. Nutr. Phys. Act. 2015, 12, 45. [CrossRef] [PubMed]

26. Luke, A.; Cooper, R.S. Physical activity does not influence obesity risk: Time to clarify the public health message. Int. J. Epidemiol. 2013, 42, 1831-1836. [CrossRef]

27. Dugas, L.R.; Harders, R.; Merrill, S.; Ebersole, K.; Shoham, D.A.; Rush, E.C.; Assah, F.K.; Forrester, T.; Durazo-Arvizu, R.A.; Luke, A. Energy expenditure in adults living in developing compared with industrialized countries: A meta-analysis of doubly labeled water studies. Am. J. Clin. Nutr. 2011, 93, 427-441. [CrossRef]

28. Vandevijvere, S.; Chow, C.C.; Hall, K.D.; Umali, E.; Swinburn, B.A. Increased food energy supply as a major driver of the obesity epidemic: A global analysis. Bull. World Health Organ. 2015, 93, 446-456. [CrossRef]

29. Blair, S.N.; Archer, E.; Hand, G.A. Commentary: Luke and Cooper are wrong: Physical activity has a crucial role in weight management and determinants of obesity. Int. J. Epidemiol. 2013, 42, 1836-1838. [CrossRef]

30. Wiklund, P. The role of physical activity and exercise in obesity and weight management: Time for critical appraisal. J. Sport Health Sci. 2016, 5, 151-154. [CrossRef]

31. Piirtola, M.; Kaprio, J.; Waller, K.; Heikkilä, K.; Koskenvuo, M.; Svedberg, P.; Silventoinen, K.; Kujala, U.M.; Ropponen, A. Leisure-time physical inactivity and association with body mass index: A Finnish Twin Study with a 35-year follow-up. Int. J. Epidemiol. 2017, 46, 116-127. [CrossRef]

32. Pendergast, F.J.; Livingstone, K.M.; Worsley, A.; McNaughton, S.A. Examining the correlates of meal skipping in Australian young adults. Nutr. J. 2019, 18, 24. [CrossRef]

33. Munt, A.E.; Partridge, S.R.; Allman-Farinelli, M. The barriers and enablers of healthy eating among young adults: A missing piece of the obesity puzzle: A scoping review. Obes. Rev. 2017, 18, 1-17. [CrossRef] [PubMed]

34. Ministerio de Sanidad Servicios Sociales e Igualdad; Instituto Nacional de Estadística. Encuesta Europea de Salud en España EESE 2009; Madrid, Ministerio de Sanidad Servicios Sociales e Igualdad. 2010. Available online: www.ine.es (accessed on 6 January 2019).

35. Ministerio de Sanidad Servicios Sociales e Igualdad; Instituto Nacional de Estadística. Encuesta Europea de Salud en España EESE 2014; Madrid, Ministerio de Sanidad Servicios Sociales e Igualdad. 2015. Available online: www.ine.es (accessed on 6 January 2019).

36. Ministerio de Sanidad Servicios Sociales e Igualdad; Instituto Nacional de Estadística. Encuesta Nacional de Salud España ENSE 2011/12; Madrid, Ministerio de Sanidad Servicios Sociales e Igualdad. 2013. Available online: www.ine.es (accessed on 9 January 2019).

37. Ministerio de Sanidad Servicios Sociales e Igualdad; Instituto Nacional de Estadística. Encuesta Nacional de Salud España ENSE 2017; Madrid, Ministerio de Sanidad Servicios Sociales e Igualdad. 2018. Available online: www.ine.es (accessed on 9 January 2019).

38. Domingo-Salvany, A.; Bacigalupe, A.; Carrasco, J.M.; Espelt, A.; Ferrando, J.; Borrell, C. Propuestas de clase social neoweberiana y neomarxista a partir de la Clasificación Nacional de Ocupaciones 2011. Gac. Sanit. 2013, 27, 263-272. [CrossRef] [PubMed]

39. Sander, C.; Ueck, P.; Mergl, R.; Gordon, G.; Hegerl, U.; Himmerich, H. Physical activity in depressed and non-depressed patients with obesity. Eat Weight Disord-Stud Anorexia. Bulim Obes. 2018, 23, 195-203.

40. WHO. Depresion. World Health Organization. 2018. Available online: https://www.who.int/en/news-room/ fact-sheets/detail/depression (accessed on 15 July 2019).

41. Ball, K.; Burton, N.W.; Brown, W.J. A Prospective Study of Overweight, Physical Activity, and Depressive Symptoms in Young Women. Obesity 2009, 17, 66-71. [CrossRef] 
42. Bjerkeset, O.; Romundstad, P.; Evans, J.; Gunnell, D. Association of Adult Body Mass Index and Height with Anxiety, Depression, and Suicide in the General Population: The HUNT Study. Am. J. Epidemiol. 2007, 167, 193-202. [CrossRef]

43. Rhew, I.C.; Richardson, L.P.; Lymp, J.; McTiernan, A.; McCauley, E.; Vander Stoep, A. Measurement matters in the association between early adolescent depressive symptoms and body mass index. Gen. Hosp. Psychiatry 2008, 30, 458-466. [CrossRef]

44. Blough, J.; Loprinzi, P.D. Experimentally investigating the joint effects of physical activity and sedentary behavior on depression and anxiety: A randomized controlled trial. J. Affect. Disord. 2018, 239, $258-268$. [CrossRef]

45. Bailey, A.P.; Hetrick, S.E.; Rosenbaum, S.; Purcell, R.; Parker, A.G. Treating depression with physical activity in adolescents and young adults: A systematic review and meta-analysis of randomised controlled trials. Psychol. Med. 2018, 48, 1068-1083. [CrossRef]

46. Dankel, S.J.; Loenneke, J.P.; Loprinzi, P.D. Mild Depressive Symptoms among Americans in Relation to Physical Activity, Current Overweight/Obesity, and Self-Reported History of Overweight/Obesity. Int. J. Behav. Med. 2016, 23, 553-560. [CrossRef]

47. Wilks, D.C.; Sharp, S.J.; Ekelund, U.; Thompson, S.G.; Mander, A.P.; Turner, R.M.; Jebb, S.A.; Lindroos, A.K. Objectively Measured Physical Activity and Fat Mass in Children: A Bias-Adjusted Meta-Analysis of Prospective Studies. PLoS ONE 2011, 6, e17205. [CrossRef]

48. Sayegh, S.; Van Der Walt, M.; Al-Kuwari, M.G. One-Year assessment of physical activity level in adult Qatari females: A pedometer-based longitudinal study. Int. J. Womens Health 2016, 8, 287-293. [CrossRef] [PubMed]

49. Joseph, R.P.; Royse, K.E.; Benitez, T.J.; Pekmezi, D.W. Physical activity and quality of life among university students: Exploring self-efficacy, self-esteem, and affect as potential mediators. Qual. Life Res. 2014, 23, 659-667. [CrossRef] [PubMed]

50. Murphy, M.H.; Carlin, A.; Woods, C.; Nevill, A.; MacDonncha, C.; Ferguson, K.; Murphy, N. Active Students Are Healthier and Happier Than Their Inactive Peers: The Results of a Large Representative Cross-Sectional Study of University Students in Ireland. J. Phys. Act. Health 2018, 15, 737-746. [CrossRef] [PubMed]

51. Laredo-Aguilera, J.A.; Carmona-Torres, J.M.; Cobo-Cuenca, A.I.; García-Pinillos, F.; Latorre-Román, P.A. Handgrip Strength is Associated with Psychological Functioning, Mood and Sleep in Women over 65 Years. Int. J. Environ. Res. Public Health 2019, 16, 873. [CrossRef] [PubMed]

52. Pelusi, C.; Altieri, P.; Gambineri, A.; Repaci, A.; Cavazza, C.; Fanelli, F.; Morselli-Labate, A.M.; Pagotto, U.; Pasquali, R. Behavioral, socio-environmental, educational and demographic correlates of excess body weight in Italian adolescents and young adults. Nutr. Metab. Cardiovasc. Dis. 2019, 29, 279-289. [CrossRef] [PubMed]

53. Chung, A.; Backholer, K.; Wong, E.; Palermo, C.; Keating, C.; Peeters, A. Trends in child and adolescent obesity prevalence in economically advanced countries according to socioeconomic position: A systematic review. Obes. Rev. 2016, 17, 276-295. [CrossRef]

54. Balhareth, A.; Meertens, R.; Kremers, S.; Sleddens, E. Overweight and obesity among adults in the Gulf States: A systematic literature review of correlates of weight, weight-related behaviours, and interventions. Obes. Rev. 2019, 20, 763-793. [CrossRef]

55. Peterson, N.E.; Sirard, J.R.; Kulbok, P.A.; DeBoer, M.D.; Erickson, J.M. Sedentary behavior and physical activity of young adult university students. Res. Nurs. Health 2018, 41, 30-38. [CrossRef]

56. Monteiro, L.Z.; Varela, A.R.; de Lira, B.A.; Contiero, L.C.; Carneiro, M.D.; de Souza, P.; de Toledo Nóbrega, J.O.; Júnior, F.B. Weight status, physical activity and eating habits of young adults in Midwest Brazil. Public Health Nutr. 2019, 22, 2609-2616. [CrossRef]

57. Dodge, T.; Clarke, P.; Dwan, R. The Relationship between Physical Activity and Alcohol Use among Adults in the United States. Am. J. Health Promot. 2017, 31, 97-108. [CrossRef]

58. Rowland, B.C.; Wolfenden, L.; Gillham, K.; Kingsland, M.; Richardson, B.; Wiggers, J. Is alcohol and community sport a good mix? Alcohol management, consumption and social capital in community sports clubs. Aust. N. Z. J. Public Health 2015, 39, 210-215. [CrossRef] [PubMed]

59. Stang, J.; Bonilla, Z. Factors Affecting Nutrition and Physical Activity Behaviors of Hispanic Families with Young Children: Implications for Obesity Policies and Programs. J. Nutr. Educ. Behav. 2018, 50, 959-967. [CrossRef] [PubMed] 
60. Flores Mateo, G.; Granado-Font, E.; Ferré-Grau, C.; Montaña-Carreras, X. Mobile Phone Apps to Promote Weight Loss and Increase Physical Activity: A Systematic Review and Meta-Analysis. J. Med. Internet Res. 2015, 17, e253. [CrossRef] [PubMed]

61. Quan, M.; Pope, Z.; Gao, Z. Examining Young Children's Physical Activity and Sedentary Behaviors in an Exergaming Program Using Accelerometry. J. Clin. Med. 2018, 7, 302. [CrossRef]

62. Zeng, N.; Pope, Z.; Lee, J.; Gao, Z. Virtual Reality Exercise for Anxiety and Depression: A Preliminary Review of Current Research in an Emerging Field. J. Clin. Med. 2018, 7, 42. [CrossRef]

(C) 2019 by the authors. Licensee MDPI, Basel, Switzerland. This article is an open access article distributed under the terms and conditions of the Creative Commons Attribution (CC BY) license (http://creativecommons.org/licenses/by/4.0/). 